\section{Dermoscopy is a new diagnostic tool in diagnosis of common hypopigmented macular disease: A descriptive study}

\author{
Khitam Al-Refu \\ Faculty of Medicine, Mutah University, \\ Jordan
}

\begin{abstract}
One of the most frequent complaints in dermatology clinics is the eruption of hypopigmented patchy skin lesions. The aim of the study was to investigate the utility of dermoscopy in common hypopigmented macular diseases. Patients with the followings diseases were examined by dermoscopy: vitiligo, pityriasis alba, nevus depigmentosus, achromic pityriasis versicolor, idiopathic guttate hypomelanosis, and extragenital guttate lichen sclerosus.

This study showed that these hypopigmented macular diseases might display specific dermoscopic features. In vitiligo, the mean dermoscopic features were the presence of a diffuse white glow with perifollicular pigment, perilesional hyperpigmentation, leukotrichia and the pigmentary network. In idiopathic guttate hypomelanosis, the characteristic features were the presence of multiple, shiny, scaly macules with welland ill-defined edges borders that coalesced into polycyclic macules. For nevus depigmentosus, the mean features were hypopigmented patches with irregular border with a faint reticular network. For pityriasis alba, the fairly ill-demarcated hypopigmented macules with fine scales were the mean feature. In lichen sclerosus, there were white structureless areas, perilesional erythematous halo, follicular plugging and white chrysalis like structures. Dermoscopy of achromic pityriasis versicolor showed a fairly demarcated white area with fine scales localized in the skin creases.
\end{abstract}

\section{Introduction}

Over the last few years, dermoscopy has been shown to be a helpful tool in the diagnosis of many general dermatological disorders. ${ }^{1-7}$ In addition to its well-documented value in the diagnosis of skin tumors and pigmentary melanocytic lesions, dermoscopy is very helpful in assisting the diagnosis of various general dermatological disorders, including scalp/hair diseases, ${ }^{8}$ nail/ nail-fold abnormalities, ${ }^{9}$ and inflammatory dermatoses. ${ }^{1}$
One of the commonest complaints in dermatology clinics is the eruption of macular or patchy hypopigmented skin lesions. This complaint may be very disturbing to the patient, especially for those with dark skin. Some of important hypopigmented macular disease $^{1}$ are vitiligo, pityriasis alba, extragenital lichen sclerosus, achromic pityriasis versicolor, idiopathic guttate hypomelanosis and nevus depigmentosus. Generally, these disorders share the same patient's complaint which is characterized by the presence of hypo or depigmented patches or macules. In this study, the aim was to provide an overview on the use of dermoscopy in dermatology by analyzing the dermoscopic features of some of important hypopigmented macular diseases. New studies have documented dermoscopic features in vitiligo. ${ }^{10-19}$ Very few reports documented these changes in idiopathic guttate hypomelanosis ${ }^{20,21}$ nevus depigmentosus, ${ }^{22-24}$ achromic pityriasis versicolor ${ }^{25}$ and extragenital lichen sclerosus. ${ }^{26-28}$ This study investigated 108 patients presented at dermatology clinics during the last three years complaining of hypopigmented and depigmented cutaneous lesions. This current study contained a larger number of cases, aiming to describe the common and uncommon dermoscopic features for the hypopigmented macular disease; comparing the findings with the previous reports and documenting the frequency of them.

\section{Materials and Methods}

A total of 108 patients were seen at the dermatology clinics (Mutah University Medical Center) during January 2015 to October 2017 complaining of patchy or macular hypopigmented lesions. Detailed and informed consent was taken from the patients. The sample collection was not consecutive, as the intention was to investigate the patients who were diagnosed with specific hypopigmented skin diseases. The following skin diseases were included in the research: vitiligo, pityriasis alba, nevus depigmentosus, achromic pityriasis versicolor, idiopathic guttate hypomelanosis, and extragenital guttate lichen sclerosus. All of the cases which were on treatment were excluded from the study. This was of particular concern for the cases of vitiligo; the patients were on no treatment for three months prior investigations. A detailed history was taken, and dermatological examination was done. A histopathological examination was done for all cases of idiopathic guttate hypomelanosis and lichen sclerosus et atrophicus to confirm the diagnosis of the disease. The histology was not performed
Correspondence: Khitam Al-Refu, Faculty of Medicine, Mutah University, Jordan.

Tel.: 00962797401149.

E-mail: alrefukhi@yahoo.com alrefukhi@mutah.edu.jo

Key words: Dermoscopy; Diagnosis; Hypopigmented macules; Vitiligo.

Conflict of interest: the author declares no potential conflict of interest.

Funding: none.

Received for publication: 26 October 2018.

Revision received: 28 November 2018.

Accepted for publication: 29 November 2018

This work is licensed under a Creative Commons Attribution-NonCommercial 4.0 International License (CC BY-NC 4.0).

(C) Copyright K. Al-Refu, 2018

Licensee PAGEPress, Italy

Dermatology Reports 2019; 11:7916

doi:10.4081/dr.2018.7916

for other hypopigmented skin diseases as the diagnosis was based on clinical background combined with Wood's light examination and potassium hydroxide $(\mathrm{KOH})$ scrapping. All of the cases were underwent Wood's light examination. This was a very crucial method to confirm the diagnosis of some cases such as vitiligo and pityriasis versicolor. All of the scaly macules and patches were examined with potassium hydroxide scrapping. This is a very important test to confirm the diagnosis of all suspected cases of pityriasis versicolor. The gently scrapped skin scales were placed directly onto a microscope slide and are covered with $10 \%$ potassium hydroxide, and were left to stand until clear and gently heated to speed up the action of $\mathrm{KOH}$. A dermoscopic examination of all cases was performed using a hand-held dermoscope (Dermlite DL3, Gen, USA) application (10× magnification) with both polarized and non-polarized lights. iPhone camera was attached to capture images. The dates and times of capturing photos were automatically stored. The dermoscopic examination was done for all cases looking for specific diagnostic finding for each case. The dermoscopic examination was done blindly without referring to the clinical or histological information. The following parameters were taken into consideration when applying dermoscopy in these diseases: i) presence of altered pigmentation within the patch or macule; ii) The edge of the macules or patch; whether it is fairly demarcated or ill-defined; iii) Presence of 
the scales within the lesion or surrounding skin; iv) Presence of perifollicular pigmentation; v) Presence of perilesional reticulated hyperpigmentation or telangiectasia; vi) The color of the hair within the hypopigmented lesions; vii) The morphology/ arrangement of vascular structures. Regarding the pattern or the shape of macules in dermoscopy, there were different patterns described included: ${ }^{10}$ trichrome pattern, nebulous pattern, polka dot pattern (several depigmented macules), comet tailing of the lesion, amoeboid pattern, and petaloid pattern.

\section{Results}

In this study, 108 patients with the followings diseases were enrolled in the study: vitiligo (48 cases), pityriasis alba (16 cases), nevus depigmentosus (8 cases), achromic pityriasis versicolor (16 cases), idiopathic guttate hypomelanosis (11 cases), and extragenital guttate lichen sclerosus (9 cases). Out of 48 patients with vitiligo included in the study, 25 were females, and 23 were males. The duration of the disease ranged from 8 months to 2 years; with an average duration of 4 months. The mean age of onset was 24 years. Among the patterns of vitiligo, 23 patients had vitiligo vulgaris, acral distribution in 11 cases, a focal variant in 10 cases and 4 had segmental distribution. The most common site was extremities followed by trunk and face. All cases demonstrated bright white (depigmented) areas with Wood's light illumination with bright bluewhite fluorescence. The dermoscopic analysis was done for established, progressive, or regimenting vitiligo. All of the cases showed different activity of the disease even within the same patient. Regarding the pattern of pigmentation dermoscopy, a diffuse white glow was seen in $78 \%$ of cases, perilesional hyperpigmentation was present in $30 \%$ of cases, perifollicular hyperpigmentation was present in $75 \%$ of cases, interfollicular pigmentation was seen in $40 \%$ of cases, white villus and terminal hair in $70 \%$ of cases, the pigmentary network within the lesions in $23 \%$ of cases, lesional and perilesional telangiectasia were present in $8 \%$ of cases. All of the lesions were not scaly in this study. Regarding the pattern in dermoscopy, there were different patterns seen in this study in order of frequency as follows: nebulous pattern ( $72 \%$ of lesions), amoeboid pattern (63\% of lesions), trichrome pattern ( $58 \%$ of lesions), petaloid pattern (51\% of lesions), polka dot pattern (46\% of lesions) and comet tailing of the lesion ( $28 \%$ of lesions). Figure 1 demonstrates some of these dermoscopic features in vitiligo patients.

Patients with idiopathic guttate hypomelanosis were 11 cases $(7$ males, four females). The Average age of the patients was 55.3 years with a range of 35 years to 80 years. On physical examination, there were multiple circular hypopigmented macules of various sizes ranging from 1 to 3 $\mathrm{mm}$ in diameter. The skin was scaly and showed some features of sun-damage. Average disease duration was 6 years. The histopathological findings for skin biopsies from these lesions demonstrated basketwave hyperkeratosis, epidermal atrophy, and flattened rete ridges, and reduction of melanin pigment and numbers of melanocytes. Dermoscopic features (Figure 2A) for these cases were nearly similar in all cases and was characterized by the presence of multiple, shiny, porcelain-white macules with well- and ill-defined edges borders. These macules may coalesce into polycyclic macules. Hyperpigmented networks may be seen within the lesions or surrounding skin giving the appearance of the cloudy-sky pattern. The surrounding skin was scaly. The hair was pigmented with no peri-follicular hyperpigmentation. In addition, as has been described in vitiligo, it was common to see the petaloid, amoeboid, and nebuloid and polka dot appearance in some cases (even in the same patient and in the same dermoscopic view) as has been described in Figure 2A.
Nevus depigmentosus was one of the common differential diagnosis of vitiligo. All of the presented cases in this research were children. Eight cases presented with an asymptomatic light patches that had been first noted in the neonatal period. The patches had been stable in shape, texture, but increasing in size with age. The mean age of presentation was 3 years with a range (1.5-12 years). Physical examination for each patient revealed a single, well-defined, hypopigmented patch, with a serrated border. The surrounding skin was normal. Wood's light examination of the patch showed an off-white accentuation without fluorescence. On dermoscopy (Figure 2B), the hypopigmented patch with an irregular and serrated boarder was the prominent feature. Some of patches showed faint reticular network within them. The borders also showed pseudopods pattern protruding into the normal skin. Hairs within the patches was of normal colors, and there was no peripheral hyperpigmentation.

Sixteen patients with pityriasis alba were examined with dermoscopy. The mean age of presentation was 8.2 years. The mean duration of the patches was 12 weeks, and no treatment was prescribed. $\mathrm{KOH}$ scraping was negative for all cases and similarly was the Wood's light examination. On dermoscopic examination (Figure 3A), the hypopigmented macules were fairly illdemarcated white area with fine scales that are commonly distributed within and outside the macules. The hair inside the patch-

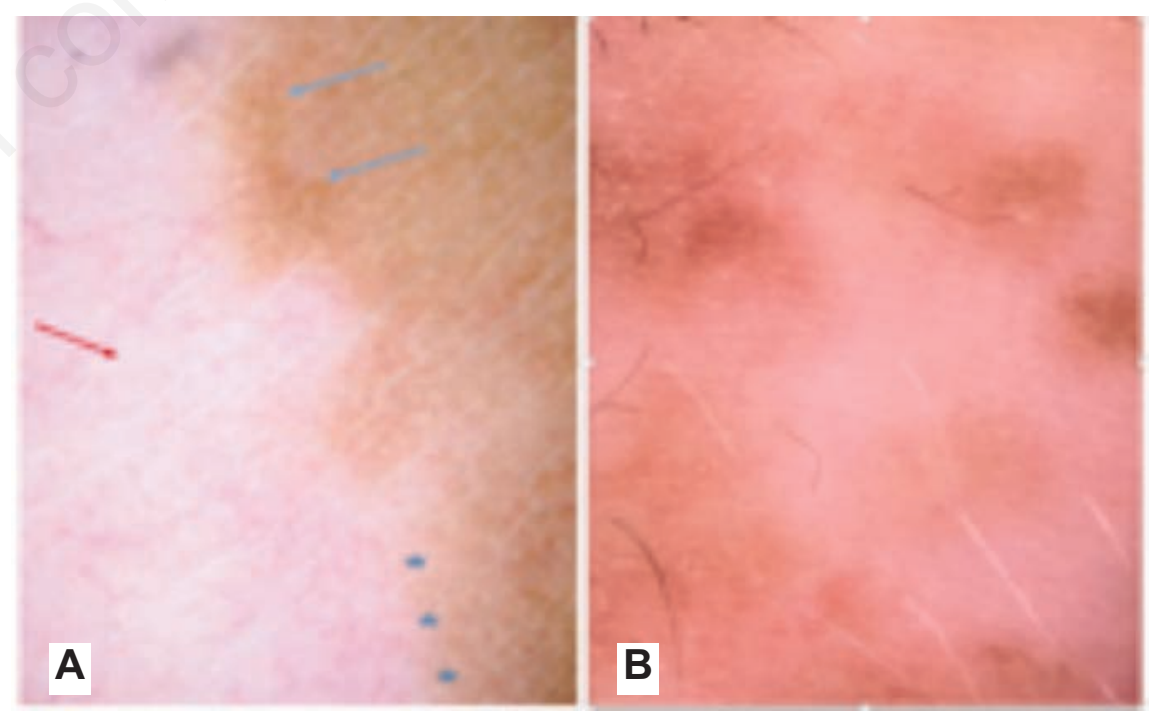

Figure 1. (A) Dermoscopic view for vitiligo show depigmented patch, perilesional hyperpigmentation (blue arrows) and depigmented hair (leukotrichia) (red arrow). It is prominent in the same view that the lower margin of the depigmented patch with ill-defined margins that merge indistinctly into the surrounding (Nebulous pattern) (blue stars). (B) Dermoscopic view for vitiligo show Polka dot appearance with several depigmented macules within a hyperpigmented background. Few macules show perifollicular repigmentation and leukotrichia as well. 
es was of normal color. There is no sharp margin to differentiate the hypopigmented patch from the surrounding skin. These features were seen in all cases of pityriasis alba. In addition, $30 \%$ of cases demonstrated erythematous changes within and surrounding the macules and patches.

All of 16 patients with pityriasis versicolor were 12 males and four females. Mean age was 23 years. They did not have treatment prior to presentation. The mean duration of disease was 9 weeks. All cases had the lesions on their necks and upper trunk. The patients were asymptomatic. The diagnosis was confirmed based on clinical assessment, $\mathrm{KOH}$ scrapping, and Wood's light illumination. The characteristic dermoscopic feature (Figure 3B) in $85 \%$ of cases was diffuse hypopigmented blotches (fairly defined) with fine scales that were commonly localized in the skin furrows. In addition, some of the cases $(65 \%)$ demonstrate satellite lesions (nearby small round white globules). In $22 \%$ of cases, the hypopigmented blotches were ill-defined, but with prominent white, scaly globules. None of the cases demonstrated perilesional hyperpigmentation or perifollicular hyperpigmentation. The hair inside these macules was of normal color but covered by scales in some cases.

In this study, 9 cases with extragenital lichen sclerosus were examined with dermoscopy. Seven females with mean age 11 years, with a range 7-18 years. Two cases are males, of age 28 and 34 years. The mean duration of the disease 1,8 years (with a range from 1,2 to 2,3 years). The presentation of these cases was white macules over the trunk and extremities. It started as erythema, expanding gradually, and finally turned out to be a white patch. Examination revealed a well-demarcated, mild atrophic porcelain-white macules. The diagnosis for this cases was confirmed by histopathology (Figure 4) which showed hyperkeratosis, epidermal atrophy, squamatization of the basal cell layer and homogenization and hyalinosis of the upper dermis.

The predominant dermoscopic feature of lichen sclerosus (Figure 5) was whiteyellowish structureless areas and white chrysalis like structures. This whitish color is glowing white and surrounded by an erythematous halo (as delicate linear branching vessels with different lengths and calibers) especially in active lesions. Fine whitish scales were prominent. Follicular plugging was seen in new lesions of lichen sclerosus et atrophicus and was not started on treatment yet. Table 1 summarized all the previous dermoscopic features in all examined hypopigmented macular diseases.


Figure 2. (A) Dermoscopy of a lesion of idiopathic guttate hypomelanosis shows multiple, roundish, homogenous, white-porcelain macules, and two were well defined (amoeboid pattern) (red arrows) and with irregular cloudy whitish pattern (nebuloid) (blue arrow). Several white shades present within the field represents the scales. In addition, these whitish areas were surrounded by a patchy hyperpigmented network (cloudy- sky pattern) (blue stars). (B) The hypopigmented patches of nevus depigmentosus with irregular border (serrated) and with a faint reticular network within the patch.
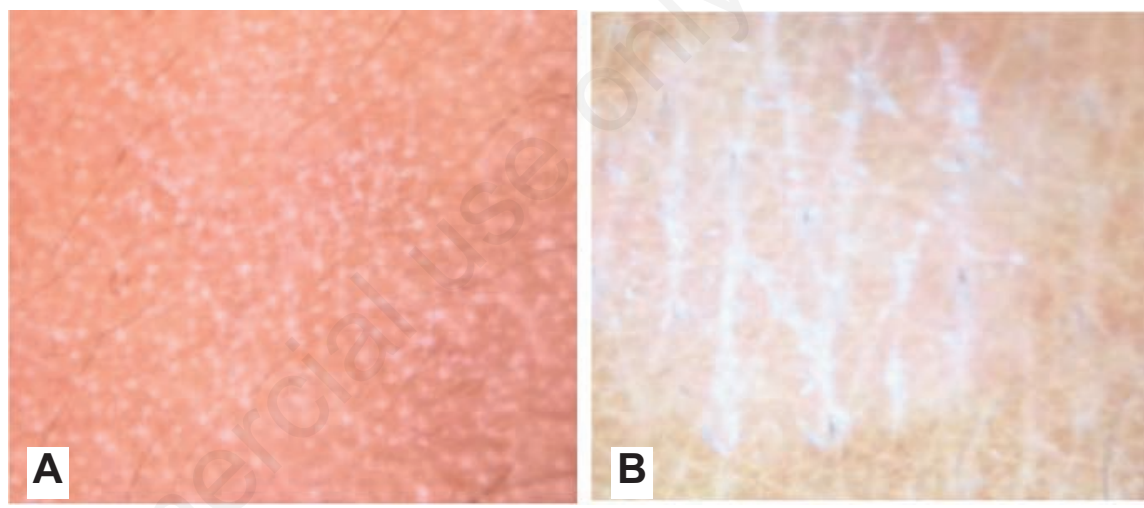

Figure 3. (A) Dermoscopic feature for a child with pityriasis alba. The hypopigmented macules were fairly ill-demarcated white area with fine scales that are commonly distributed within and outside the macules. The hair inside the patches was of normal color. There is no sharp margin to differentiate the hypopigmented patch from the surrounding skin. (B) Dermoscopic view for achromic pityriasis versicolor showed a fairly demarcated white area with fine scales that are commonly localized in the skin creases.

\section{Discussion}

Hypomelanosis of the skin encompasses a wide spectrum of congenital and acquired alterations in melanin pigmentation. They pose a diagnostic challenge for the clinician; many of these hypopigmented skin lesions appear similar. Vitiligo is one of the most common forms of acquired hypomelanosis of the skin which can be treated in a guideline-oriented manner. But it carries tremendous social implications as social stigma, especially in Arab countries and one of these Jordan (this research has been done). The eruption of hypopigmented skin lesions is of particular concern especially in darker skin patients. The common skin type in Arab countries is the type III and IV Fitzpatrick skin types. Therefore,

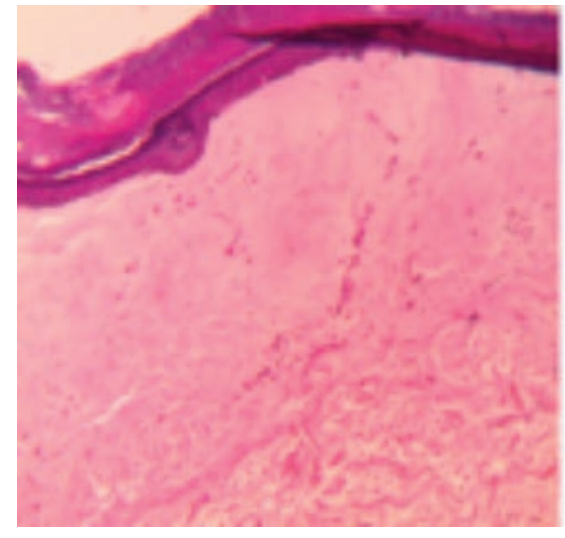

Figure 4. Histopathology of a lesion of extragenital lichen sclerosus showed hyperkeratosis, epidermal atrophy, squamatization of the basal cell layer and homogenization and hyalinization of the upper dermis. 
there is a need for standardized criteria to differentiate this disease from other hypopigmented macular diseases. The dermoscopy may be a helpful as a non-invasive tool in assisting the differential diagnosis of several hypopigmented macular lesions and has the potential to improve the diagnostic accuracy.

Vitiligo is an acquired, autoimmune disorder characterized by well-demarcated depigmented macules and patches with or without leukotrichia. Evolving new lesions of vitiligo are difficult to be distinguished clinically from other causes of hypopigmentation and depigmentation. The dermoscopic examination can detect subtle changes which may be useful in the early diagnosis of vitiligo from other differential diagnoses of vitiligo. Some workers have studied the utility of dermoscopy in the diagnosis of vitiligo. A pattern of depigmentation with residual reservoirs of perifollicular pigment have been noted, and this was signifying focally active or regimenting vitiligo. ${ }^{15}$ This residual perifollicular pigmentation had been observed in $(91.9 \%)$ of patients with progressing vitiligo and $(62.9 \%)$ of those with stable vitiligo, ${ }^{16}$ and this can be considered as characteristic for vitiligo lesions as it was absent in the nonvitiligo depigmented skin. ${ }^{16}$ In this study, 48 cases of vitiligo had been examined by dermoscopy. A diffuse white glow was seen in $78 \%$ of cases, perilesional hyperpigmentation was present in $30 \%$ of cases, and perifollicular hyperpigmentation was present in $75 \%$ of cases. In this study, the dermoscopic analysis was done for established, progressive, or regimenting vitiligo, and this may explain the difference in frequency from the



previous study. In addition, interfollicular pigmentation was seen in $40 \%$ of cases, white villus and terminal hair in $70 \%$ of cases, the pigmentary network within the lesions in $23 \%$ of cases and lesional and perilesional telangiectasia were present in $8 \%$ of cases. The presence of telangiectasia may be related to the history of treatment of patients by topical steroids. This marginal and perifollicular marginal and perifollicular hyperpigmentation, reticular pigmentation and marginal reticular pigmentation may be associated with stability and repigmentation of vitiligo. ${ }^{17}$ Since the progressive lesions of vitiligo display perifollicular pigmentation and stable/remitting lesions display perifollicular depigmentation. ${ }^{18}$ Regarding the pattern, there were different patterns seen in order of frequency as follows: nebulous pattern ( $72 \%$ of lesions), amoeboid pattern (63\% of lesions), trichrome pattern (58\% of lesions), petalloid pattern ( $51 \%$ of lesions), polka dot pattern (46\% of lesions) and comet tailing of the lesion ( $28 \%$ of lesions). A similar pattern has been reported before. ${ }^{10}$

\section{Conclusions}

In conclusion, this study confirmed the findings in previous studies; the glowing white color was characteristic for vitiligo. In addition, this perifollicular pigmentation was seen in all stable and progressive cases of vitiligo. There was a different pattern for the depigmented patch of vitiligo, and this may reflect the activity and the progression of the disease.

Idiopathic guttate hypomelanosis is

another disease characteristically presented 


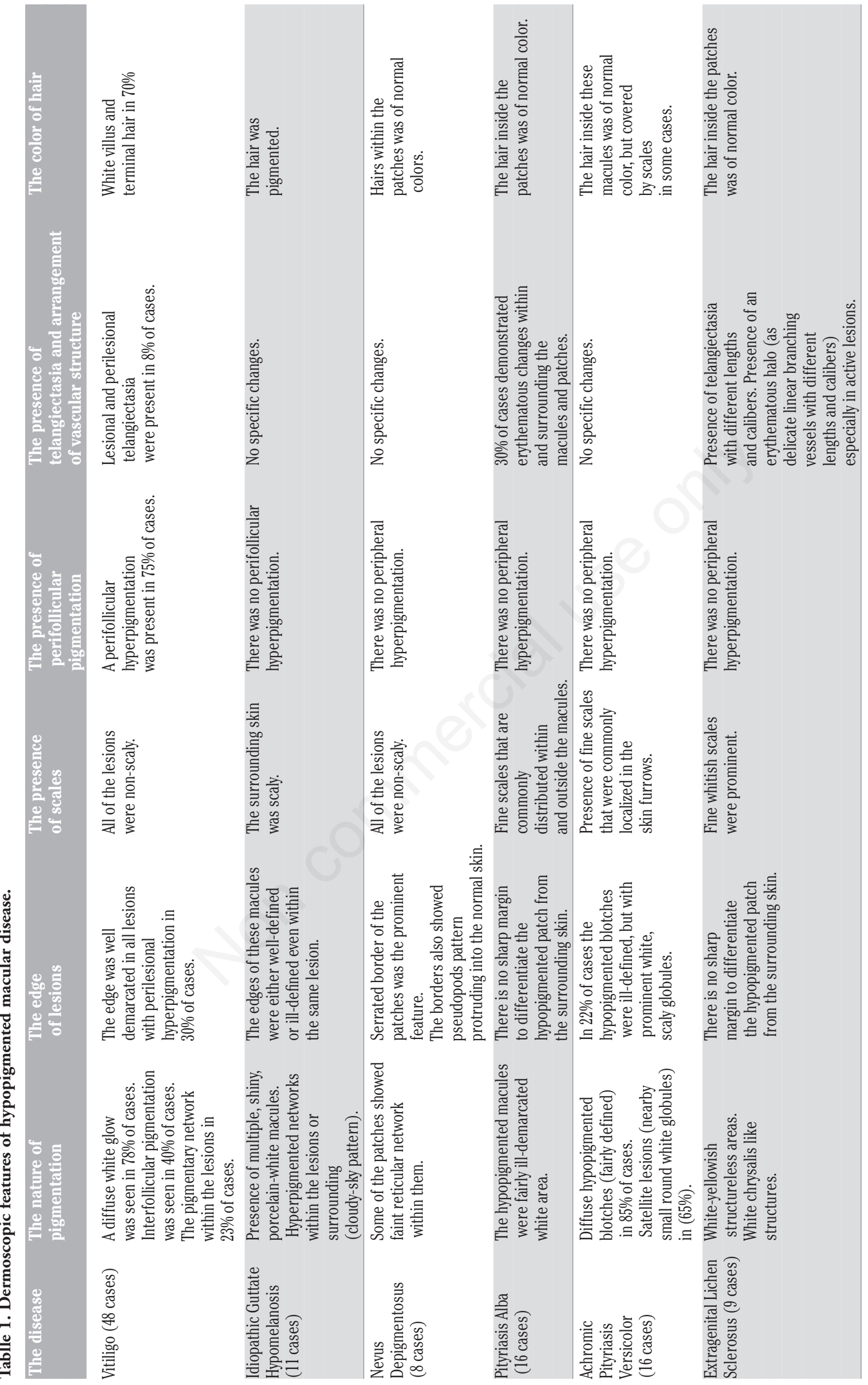


the normal skin. Hair within the patches was of normal colors, and there was no peripheral hyperpigmentation. In addition, there was a normal colored peri-lesional skin. This feature was described before. ${ }^{23,24}$

Pityriasis alba is a common benign skin condition causing hypopigmented facial patches in children. Parents and patients are often concerned about the cosmetic appearance and the similarity to the lesions of vitiligo (from the community view). But here, the patches tend to be ill-defined. In addition, Wood's light has little effect on the hypopigmentation of pityriasis alba. Sixteen cases with pityriasis alba were examined with dermoscopy. One previous report investigated this disease by dermoscopy. ${ }^{25}$ On dermoscopic examination, the hypopigmented macules were fairly illdemarcated white area with fine scales that are commonly distributed within and outside the macules. The hair inside the patches was of normal color. There is no sharp margin to differentiate the hypopigmented patch from the surrounded skin. These features were seen in all cases of pityriasis alba. In addition, $30 \%$ of cases demonstrated erythematous changes within and surrounding the macules and patches.

In this study, all of 16 patients with pityriasis versicolor were examined by dermoscopy. The characteristic dermoscopic feature in $78 \%$ of cases was diffuse hypopigmented blotches (fairly defined) with fine scales that were commonly localized in the skin furrows. In addition, most of the cases $(85 \%)$ demonstrate satellite lesions (nearby small round white globules). In $22 \%$ of cases, the hypopigmented blotches were ill-defined, but with prominent white, scaly globules. Actually, this dermoscopic feature of presence of scales that were localized in the skin furrows was characteristic to pityriasis versicolor and was not seen in other diseases that have been studied here.

Extra-genital lichen sclerosus et-atrophicus is a chronic inflammatory dermatosis of unknown etiology. The presentation of these cases was with white macules over the trunk and extremities. It started as erythema, expanding gradually, and finally turned out to be a white patch. Examination revealed a well-demarcated, mild atrophic porcelain-white macules. These cases were examined with dermoscopy, the predominant dermoscopic feature of lichen sclerosus is white-yellowish structureless areas, this whitish color is glowing white, and surrounded by an erythematous halo especially in active lesions. In addition, these active lesions showed follicular plugging which was absent from old lesions, and this was in correlation with a previous studies. ${ }^{26,27}$ Fine whitish scales were prominent. In addition to the presence of shiny, fibrotic, white beams radiating from the center of the lesions toward the periphery, they are described as white chrysalis like structures in previous reports. ${ }^{26,27}$ They are parallel or orthogonal or disordered linear streaks and usually seen in dermatofibroma, basal cell carcinoma, Spitz nevus and melanoma. ${ }^{28}$ Although the diagnosis of lichen sclerosus is mainly clinically and by histopathology, in early stages of the disease, both are uncharacteristic. ${ }^{29,30}$ It has been reported that histopathological changes were nonspecific in one-third of men with characteristic clinical signs of lichen sclerosus. This disease is a scarring disease, and prompt diagnosis is mandatory. These dermoscopic features were highly characteristic and not demonstrated in other hypopigmented macular lesions.

This study showed that these hypopigmented macular diseases might display specific and characteristic dermoscopic features. As described in this research, some of the dermoscopic findings in these hypopigmented macular diseases were non-specific. So, these dermoscopic findings should always be interpreted within the overall clinical context of the patient history, integrated with information from the history and the macroscopic examination., ${ }^{2,3}$ Dermoscopy may scores over routine histopathology in the diagnosis of some skin disease in which skin biopsy may be traumatic as in case of nevus depigmentosus and pityriasis alba. It's an additive test to confirm the diagnosis of pityriasis versicolor, lichen sclerosus et atrophicus and idiopathic guttate hypomelanosis.

\section{References}

1. Errichetti E, Stinco G. The practical usefulness of dermoscopy in general dermatology. J Ital Dermatol Venereol 2015;150:533-46.

2. Lallas A, Giacomel J, Argenziano G, et al. Dermoscopy in general dermatology: practical tips for the clinician. Br J Dermatol 2014;170:514-26.

3. Lallas A, Zalaudek I, Argenziano G, et al. Dermoscopy in general dermatology. Dermatol Clin 2013;31:679-94.

4. Errichetti E, Stinco G. Dermoscopy in general dermatology: a practical overview. Dermatol Ther (Heidelb) 2016;6:471-507.

5. Zalaudek I, Argenziano G, Di Stefani A, Ferrara G, et al. Dermoscopy in general dermatology. Dermatology 2006;212:718.

6. Micali G, Lacarrubba F, Massimino D,
Schwartz RA. Dermatoscopy: alternative uses in daily clinical practice. J Am Acad Dermatol 2011;64:1135-46.

7. Zalaudek I, Lallas A, Moscarella E, et al. The dermatologist's stethoscope-traditional and new applications of dermoscopy. Dermatol Pract Concept 2013;3:67-71.

8. Miteva M, Tosti A. Hair and scalp dermatoscopy. J Am Acad Dermatol 2012;67:1040-8.

9. Lencastre A, Lamas A, Sa D, Tosti A. Onychoscopy. Clin Dermatol 2013;31: 587-93.

10. Gandhi S, Shamanur M, Shashikiran AR, et al. Study of clinico-epidemiological and dermoscopic patterns of vitiligo in pediatric age group. Ind J Paediatr Dermatol 2017;18:292-8.

11. Alghamdi KM, Kumar A, Taïeb A, Ezzedine K. Assessment methods for the evaluation of vitiligo. J Eur Acad Dermatol Venereol 2012;26:1463-71.

12. Sosa JJ, Currimbhoy SD, Ukoha U, et al. Confetti-like depigmentation: A potential sign of rapidly progressing vitiligo. J Am Acad Dermatol 2015;73:272-5.

13. Benzekri L, Gauthier Y. Clinical markers of vitiligo activity. J Am Acad Dermatol 2017;76:856-62.

14. Thatte SS, Khopkar US. The utility of dermoscopy in the diagnosis of evolving lesions of vitiligo. Ind J Der VL 2014;80:505-8.

15. Chuh AA, Zawar V. Demonstration of residual perifollicular pigmentation in localized vitiligo-a reverse and novel application of digital epiluminescence dermoscopy. Comput Med Imaging Graph 2004;28:213-7.

16. Meng R, Zhao G, Cai RK, et al. Application of polarized light dermoscopy in the early diagnosis of vitiligo and its differential diagnosis from other depigmented diseases. Chin J Dermatol 2009;42:810-3.

17. Chandrashekhar L. Dermoscopy: A tool to assess stability in Vitiligo. In: Khopkar U, ed. Dermoscopy and trichoscopy in diseases of the brown skin: atlas and short text. New Delhi, India: Jaypee Brothers Medical Publishers; 2012. pp 112-113.

18. Kumar Jha A, Sonthalia S, Lallas A, Chaudhary RKP. Dermoscopy in vitiligo: diagnosis and beyond. Int $\mathrm{J}$ Dermatol 2017;26.

19. Jackson SM, Nesbitt LT. The diagnosis. In: Jackson SM, Nesbitt LT, eds. Differential diagnosis for the dermatologist, 2nd ed. New York, NY: Springer; 2012. pp 767-768.

20. Ankad BS, Beergouder SL. 
Dermoscopic evaluation of idiopathic guttate hypomelanosis: a preliminary observation. Indian Dermatol Online J 2015;6:164-7.

21. Errichetti E, Stinco G. Dermoscopy of idiopathic guttate hypomelanosis. J Dermatol 2015;42:1118-9.

22. Coupe RL. Unilateral systematized achromic naevus. Dermatologica 1967;134:19-35.

23. Ankad BS, Shah S. Dermoscopy of nevus depigmentosus. Pigment Int 2017;4:121-3.

24. Sarma N, Akkad BS. Dermoscopy in nevoid disorders: dermoscopy in darker skins. New Delhi, India: Japee
Brothers; 2017. pp 127-128.

25. Nayak SS, Mehta HH, Gajjar PC, Nimbark VN. Dermoscopy of general dermatological conditions in Indian population: A descriptive study. Clin Derm Rev 2017;1:41-51.

26. Ankad BS, Beergouder BL. Dermoscopic patterns in lichen sclerosus: A report of three cases. Ind D J 2015;6:237-40.

27. Errichetti E, Lallas A, Apalla Z, et al. Dermoscopy of morphea and cutaneous lichen sclerosus: clinicopathological correlation study and comparative analysis. Dermatology 2017;233:46270.
28. Marghoob AA, Cowell L, Kopf AW, Scope A. Observation of chrysalis structures with polarized dermoscopy. Arch Dermatol 2009;145:618.

29. Carlson JA, Lamb P, Malfetano J, et al. Clinicopathologic comparison of vulvar and extragenital lichen sclerosus: Histologic variants, evolving lesions, and etiology of 141 cases. Mod Pathol 1998;11:844-54.

30. Edmonds EV, Oyama N, Chan I, et al. Extracellular matrix protein 1 autoantibodies in male genital lichen sclerosus. Br J Dermatol 2011;165:218-9. 